\title{
Latham's life-forms
}

William Latham is working to establish a place for computers in the world of art. He sets the design rules to be followed and his program generates images in a process that could be called a simulation of evolution in action.

\section{Martin Kemp}

$\mathrm{t}$ seems to me that there is a real problem with computer art. The problem does not reside in getting the new tool to perform old artistic tricks but rather with a more profound identification of what new territories computers and art might inhabit in terms of the inherent nature of computational procedures.

One approach, pioneered by Benoit Mandelbrot in his fractal images, has been to use iteration to model mathematical sets in visual terms. The results may be seen as 'art' — just as aesthetic status can be accorded to Renaissance drawings of the Platonic solids or to Henri Poincaré's geometrical models - but they inhabit only a limited domain in the wide territories already colonized by the visual arts.

\section{Standard computer-aided design} programs, and the kinds of software 'palettes' aimed specifically at artists, are at best seen as providing ingenious extensions to hand-driven techniques or at worst as giving lazy operatives the opportunity to exploit methods they don't understand. But a few artists are conducting a more searching exploration of art forms that can be generated only with computers.

The most promising approach, exemplified by British sculptor William Latham, is to set up programs on the basis of aesthetic choices in such a way that the parameters of style and content in the images are established but the final form is not predetermined. Indeed, a fixed 'final form' may not be the end in view. Rather, the evolutionary program gives rise to a new type of kinetic sculpture.

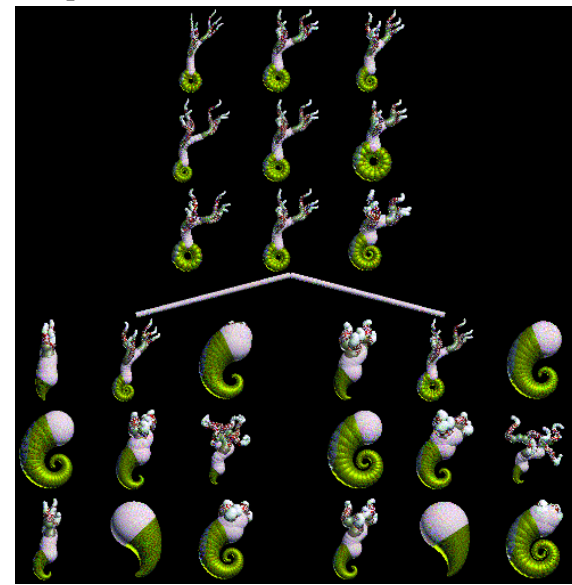

Latham's Family Tree of Evolving Forms, computer-generated using Mutator.

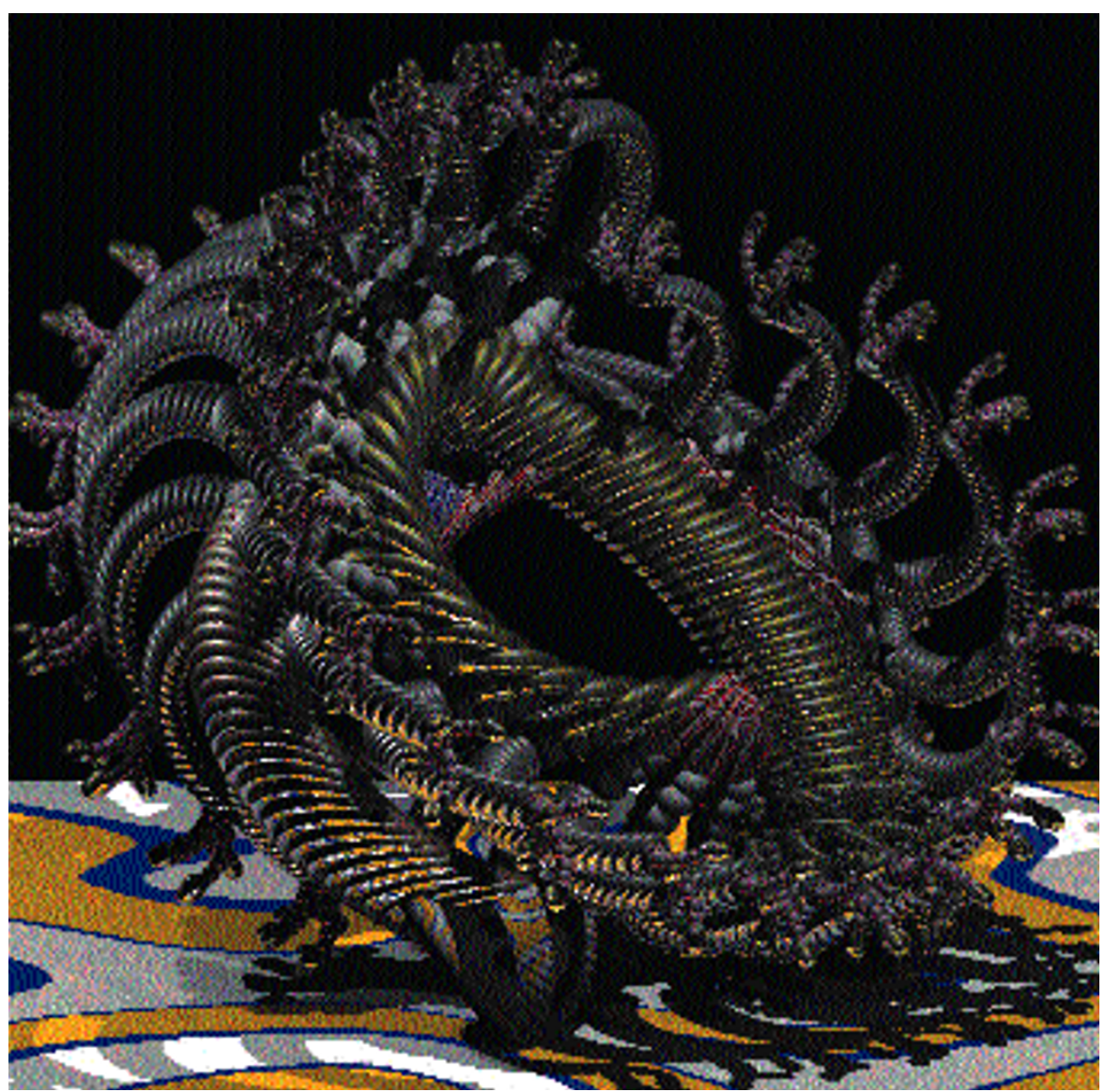

Latham's Evolving Form, computer-generated using Mutator.

Latham spent six years as research fellow in the IBM Scientific Centre at Winchester in southern England, and has developed his programs in collaboration with Stephen Todd. Most notable is a powerful and wideranging design tool called Mutator, with which sculptural forms are endowed with genetic properties that shape their growth. The basis of Mutator is provided by Form Grow, a geometrical grammar that uses spirals and fractal recursion to emulate the kinds of geometry of natural forms that have fascinated students of nature from the time of Leonardo and Dürer.

\section{Random mutation allows the artist to} navigate through the space of the infinitely varied forms that are inherent in Form Grow. The program also provides rules through which the 'lifeforms' are subject to processes of 'natural selection'. The results of such "Darwinian evolution driven by human aesthetics" are fantastical organisms whose morphologies metamorphose in a sequence of animated images.
The still images on this page show the kinds of form Latham generates, working "like a 'gardener' or 'farmer', repeatedly pidking, marrying and breeding, starting from a simple structure to evolve thousands of complex genetic variations". They are intricately wondrous in their compulsive convolutions, combining a strange beauty with an air of predatory menace.

Latham's images are typical of those generated by computer in that they seem irredeemably to have a computerized 'look' about them. There is a characteristic visual feel - just as the look of an oil painting is reliant on the properties of that particular medium.

Latham openly exploits these qualities, not least in relation to a certain kind of 'science-fiction' aesthetic that owes more than a little to the imaginative visions of the best draughtsmen of children's comics and the designers of Hollywood films. It is one of the characteristic visual modes of our age. $\square$ Martin Kemp is in the Department of the History of Art, University of Oxford, 35 Beaumont Street, Oxford OX1 2PG, UK. 